

\section{ESPERANZA DE UNA NUEVA NACIÓN}

En una calle los encuentras si miras con mucha atención, sus ojos te pueden revelar la esperanza de su corazón.

La traen en sus chamarras en sus bolsos, en su expresión, con ella, día a día se levantan antes de despuntar el sol.

Son personas que trabajan con empeño y con tesón, dejaron muy atrás su casa pero trajeron su ilusión.

Ellos llevan la añoranza de la tierra que quedó, sus costumbres las exaltan las recuerdan con amor.

Su sonrisa nos regalan su estridencia y su voz, las palabras que exclaman suenan como una canción.
Algunos cantan trova paisa otros lo hacen en Creol, unos bailan la lambada $y$ joropo en esplendor.

Así como en una danza que al caminar comenzó, llegaron con la confianza de tener un futuro mejor.

Yo veo en sus miradas porque conozco su razón, es motivo que hermana compartir su convicción.

Un niño que antes jugaba en el campo bajo el sol, salió una vez de su morada y la ciudad lo cobijó.

Sus vivencias nos embargan entendemos su clamor, porque somos camaradas ¡Nueva es nuestra nación! 


\title{
4. En la búsqueda del tío1: los haitianos en Estados Unidos de América
}

\author{
Gabriel Bidegain² \\ DOI: https://doi.org/10.5377/pdac.v16i0.10228 \\ Recibido: 10/02/2020 - Aceptado: 05/05/2020 \\ Los estadunidenses son un pueblo maravilloso, y las únicas nubes de tormenta son \\ la cuestión del negro y la terrible inmigración. \\ Max Weber ${ }^{3}$
}

\begin{abstract}
Resumen: El artículo intenta conocer el número de haitianos viviendo en Estados Unidos de América a partir de fuentes de ese país, específicamente los datos de la Oficina del Censo. Se basa en la revisión de fuentes secundarias de información. Se trata de desmitificar los relatos sobre los haitianos que en los años ochenta hablan de cuatro (4) millones en el exterior. Ninguna teoría sobre la migración internacional explica totalmente el comportamiento de los migrantes. Se estima en 643,341 los nacidos en Haití en 2017 y se desmitifica completamente las "narrativas" sobre los millones en el exterior. Y que son principalmente los más educados (particularmente mujeres) los que emigran a dicho país. Haití tiene un potencial demográfico y expulsor importante y seguirá expulsando gente "buscando al tío" a pesar de los impedimentos legales.
\end{abstract}

Palabras clave: haitianos, migrantes, Estados Unidos.

In search of an uncle: Haitians in the United States of America

\begin{abstract}
The article tries to know the number of Haitians living in the United States of America from sources in that country, specificaIly the data from the Census Bureau. It is based on the review of secondary sources of information. It is about demystifying the stories about Haitians who in the eighties spoke of four (4) million abroad. No theory of international migration fully explains the behavior of migrants. It is estimated at 643,341 those born in Haiti in 2017, and the "narratives" about millions abroad are completely demystified. And that it is mainly the most educated (particularly women) who immigrate to that country. Haiti has significant demographic and deportation potential and will continue to expel people "looking for uncle" despite legal impediments.
\end{abstract}

Keywords: haitians, migrants, United States of America.

\section{Introducción}

¿Por qué Haití y su emigración? Es un país considerado todavía rural dado que la mayoría de su población vive en ese medio (Censo 2003), al respecto se presenta toda una discusión sobre lo que es urbano. Actualmente es el país más poblado del Caribe superando a Cuba y la República Dominicana. Según la Encuesta de Morbilidad, Mortalidad y Utilización de Servicios (EMMUS - tipo DHS), en 2017, residi- rían 11,085 millones de habitantes con un crecimiento demográfico importante. Un volumen de más de 11 millones implica un potencial demográfico para que los haitianos emigren. Según la última encuesta EMMUS tipo DHS4 "En 2017, habría habido 278,862 nacimientos vivos y 97,000 muertes, por consecuencia la tasa de crecimiento anual para el período 2017-2018 se estima en 1.37\%. La proporción de sexos o la proporción de hombres en la fuerza laboral femenina es de 0.98 , lo que refleja una ligera

1 En referencia a PRICEMARS "Ainsi parla l'Oncle. Essais d'ethnographie" que decía que los haitianos tienen siempre un tío en Nueva York recomiendo su lectura y muy especialmente a SAINT - HUBERT, Francis. ... Et le Neveu Répondit Analyse Statistique de la Migration. Haïtienne aux Etats Unies (1953-2000), Trafford, Victoria, Canadá.

2 Gabriel Bidegain, Candidato a Doctor en Demografía en la Universidad Nacional de Córdoba (Argentina). Maestría en Demografía. Ex funcionario de las Naciones Unidas. Ex Embajador de Uruguay y ex consultor internacional (Asesor Político) del SG de la OEA. El autor vivió muchos años en Haití. email: gbidegain@hotmail.com

3 Citado en una carta desde Estados Unidos en diciembre de 1904 tomado de García Borrego (Garcia Borrego, 2006 Vol III \# 4).

4 DHS: Demographic Health Survey o en castellano Encuesta Demográfica de Salud responsable de obtener información sobre salud y demografía en los países en vías de desarrollo. 
sobrerrepresentación de las mujeres en la población total $(5,486,970$ hombres versus $5,598,949$ mujeres). La población haitiana es muy joven, con una edad promedio de 23 años y una esperanza de vida de 62.2 años, se estima que los menores de 15 años representan el $35.9 \%$ de la población total".

El índice de $\mathrm{Gini}^{5}$, uno de los más altos de América Latina y el Caribe, pasó del 66\% (IHSI, 2001) al 76\% (IHSI, Banco Mundial, 2012). "Después del terremoto del 12 de enero de 2012, las desigualdades sociales explotaron. La incidencia de la pobreza sigue siendo muy alta. Más de la mitad de la población (58.5\%) vive en pobreza absoluta, con menos de US $\$ 2.41$ por día, y poco menos de una cuarta parte (24\%), en pobreza extrema, con menos de US $\$ 1.23$ por día" (Banco Mundial, 2019), (ROUSSEAU, 2019, p. 23).

El Programa de las Naciones Unidas para el Desarrollo (PNUD) en su Informe de Desarrollo Humano de 2019 estima el Índice de Desarrollo Humano en 0.503 y ubica a Haití en el 169 rango sobre 190 países (PNUD, 2019). Es necesario subrayar, además, que Haití es el único país de América Latina y el Caribe que se encuentra dentro de la categoría de Desarrollo Humano Débil según dicha organización internacional.

En Haití, las remesas están en rápido crecimiento. Desde 2002 hasta el 2018 dichos fondos se multiplicaron por 7 pasando de 325 millones a 2,500 millones de dólares americanos (Rousseau, 2019, p. 81), (Orozco, 2019). Estima una proporción mayor (39\%).

¿De dónde vienen esos fondos? Rousseau señala que un tercio del PIB haitiano proviene de las remesas. Eso no resuelve el problema de la pobreza y del desarrollo de Haití (Rousseau, 2019, p. 81). Es importante indicar que como lo señala Portes que "no hay evidencia alguna de que por sí solas las remesas "desarrollen" económicamente a un país exportador de mano de obra" (Portes, 2009, p. 22).

Haití es un país que ha expulsado población y las emigraciones e inmigraciones se registran desde la época colonial cuando introdujeron a los esclavos provenientes de África o la emigración de los propie- tarios de extensiones de caña de azúcar, así como ex esclavos se dirigieron a Cuba durante o luego de la revolución haitiana ${ }^{6}$. Así mismo en el proceso regional del azúcar muchos haitianos se dirigieron a República Dominica para las zafras azucareras y luego siguieron emigrando a pesar de los cambios que se produjeron en la economía dominicana (desaparecieron prácticamente los "Bateys") dado que hubo un incremento importante de las actividades urbanas (comercio, construcción y servicios) (Bidegain G. , 2019, p. 115), en consecuencia, los migrantes haitianos se concentran cada vez más en actividades urbanas. Solo uno de cada tres está actualmente a nivel rural.

La principal corriente migratoria de los haitianos es hacia Estados Unidos de América (EUA), esta se ha presentado en diferentes olas migratorias (Saint - Hubert, 2003). Los intelectuales y de clase media emigraron durante la dictadura de ambos Duvalier (Papa doc y baby doc) y la migración menos educada luego del Golpe de Estado (1991) contra el presidente Aristide, además en los últimos tiempos se observa que hay predominio de la población femenina e instruida. Ya (Price-mars, 1928) ${ }^{7}$, en un famoso libro indicaba que cada haitiano "tenía un tío en Nueva York". De ahí el título de este artículo.

Después del terremoto del 2010, EUA abrió sus puertas a través del Estatuto de Protección Temporal (TPS en inglés) se estimaba que podrían beneficiarse entre 100,000 a 200,000 haitianos. Dicha protección temporal ha sido extendida hasta mayo del 2020. Debido a la incertidumbre algunos haitianos se fueron a Canadá con el sueño de ser aceptados, lo cual no es posible.

\section{Metodología}

La metodología implementada en el desarrollo de este artículo se base en la revisión de fuentes secundarias de información ${ }^{8}$. En primera instancia se realizó una revisión de documentos relacionados con la temática, la descripción de datos que proceden de información estadística producida principalmente por la oficina del Censo (Bureau of Census, 1970-2010), principal productor de información es-

5 Utilizado para medir la concentración de ingresos.

6 La revolución haitiana duró 13 años.

7 Considerado el principal pensador haitiano del Siglo XX.

8 En el marco de un Doctorado en Demografía en la Universidad Nacional de Córdoba (UNC) sobre migrantes haitianos en el exterior. 
tadística oficial del país ${ }^{9}$. Se trabajó con datos previos al próximo censo de población previsto para el 1 de abril de 2020. Haití, por su parte, no ha podido realizar el quinto Censo de Población y Vivienda por problemas de seguridad.

Según Ravestein "La principal causa de las migraciones son las disparidades económicas, y el móvil económico predomina entre los motivos de las migraciones", pero las dificultades legales impiden una migración hacia EUA. Según Portes "las teorías del desarrollo nacional, tanto en América Latina como en otros lugares, rara vez han prestado suficiente atención a la migración internacional" (Portes, 2007, p. 45).

\section{Discusión de resultados}

\subsection{Haitianos en el exterior ¿Dónde están ellos?}

La literatura especializada revela la existencia de cuatro grandes centros de atracción para migrantes haitianos: Estados Unidos, República Dominicana, Canadá, luego Francia y sus territorios de ultramar.

En el pasado ${ }^{10}$ usando las fuentes disponibles para ese momento, el autor (Bidegain G. , 2011) pudo establecer el mapa mundial de la migración haitiana (muy diferente al establecido por el geógrafo Georges Anglade, quien utiliza cifras redondas y sobre estimadas del número de haitianos en el mundo) que proporciona indicaciones suficientemente enriquecedoras. En dicho momento de cada $100 \mathrm{mi}-$ grantes haitianos identificados en todo el mundo: el $91.1 \%$ se establece en las Américas, especialmente en América del Norte (62.9\%) y el Caribe (25.1\%), la participación relativa de América del Sur y Central es débil; $3 \%$ y $0.1 \%$, respectivamente. Luego viene Europa con el 3\% de los migrantes que se establecen, en su mayor parte en Francia. En tercer lugar, está Asia, que absorbe alrededor del 2\%, o 1.9\% de los migrantes. En cuarto lugar, África alberga al 5\% de los migrantes haitianos $y$, en última instancia, el Medio Oriente.

La evidencia empírica muestra que es en Estados Unidos de América donde reside la más importante colonia de las personas nacidas en Haití (Bidegain G. , 2011, p. 142). Eran unos 5,000 nacidos en
Haití a principios de los años sesenta. Entre los años 1970 al 1980 se produce la mayor cantidad de migrantes nacidos en Haití, idéntico en la década siguiente, la variación porcentual irá disminuyendo. Luego del Terremoto (12 de enero 2010) ha habido una migración "solidaria" y no solo a ese país.

\section{Cuadro $\mathrm{N}^{\circ} 1$ Nacidos en Haití y residiendo en Estados Unidos de América}

\begin{tabular}{|c|c|c|}
\hline Años & Volumen & $\begin{array}{c}\text { Variación } \\
\text { porcentual }\end{array}$ \\
\hline $\mathbf{1 9 7 0}$ & 28,026 & \\
\hline $\mathbf{1 9 8 0}$ & 92,395 & $230 \%$ \\
\hline $\mathbf{1 9 9 0}$ & 225,393 & $143 \%$ \\
\hline $\mathbf{2 0 0 0}$ & 419,395 & $86.1 \%$ \\
\hline $\mathbf{2 0 1 0}$ & 559,831 & $33.5 \%$ \\
\hline
\end{tabular}

Fuente: CELADE - IMILA y US Census Bureau 1970-2010.

Para 2017 la Oficina del Censo de Estados Unidos estima en 643,341 migrantes haitianos, con un nivel de variación de más o menos 10,693 individuos. ${ }^{11}$ (Cuadro N²).

Cuadro №2 Estimación de población de origen haitiana y residiendo en Estados Unidos

\begin{tabular}{|c|c|c|}
\hline Años & Estimación & $\begin{array}{c}\text { Variación de la } \\
\text { Estimación+/- }\end{array}$ \\
\hline $\mathbf{2 0 1 0}$ & 542,091 & 9,831 \\
\hline $\mathbf{2 0 1 1}$ & 559,331 & 9,806 \\
\hline $\mathbf{2 0 1 2}$ & 572,896 & 10,550 \\
\hline $\mathbf{2 0 1 3}$ & 581,724 & 8,700 \\
\hline $\mathbf{2 0 1 4}$ & 597,685 & 10,181 \\
\hline $\mathbf{2 0 1 5}$ & 615,300 & 10,044 \\
\hline $\mathbf{2 0 1 6}$ & 631,912 & 10,386 \\
\hline $\mathbf{2 0 1 7}$ & 643,341 & 10,693 \\
\hline
\end{tabular}

Fuente: Bureau / ACS: US Census, período 2010 - 2017.

Los datos de la Oficina del Censo de Estados Unidos brindaron, además, la oportunidad de examinar ciertas características de los migrantes haitianos. En el 2000, las mujeres son mayoría entre los migrantes,

9 La posible subestimación de esta población ya ha sido estudiada por la propia oficina del Censo de EUA (US BUREAU OF CENSUS, 1992).

10 Del año 1970 al año 2000.

11 Estimación de los nacidos en Haití y son "los migrantes de toda la vida". Tener en cuenta que no es la migración dado que es difícil medir, con los instrumentos actuales, como menciona Domenach (DOMENACH, 1998). 
representan el $53 \%$ de los inmigrantes, por tanto, los hombres representan el $47 \%$. La proporción aumenta para el año 2010 a 55\% para las mujeres, en el caso de los hombres disminuye a $45 \%$ (Cuadro $\mathrm{N} \times 3)$.

Debido a la migración selectiva, los más educados, especialmente las mujeres, optan por establecerse en los Estados Unidos de América, mientras que los menos educados emigran a la República Dominicana (país fronterizo), en este último caso, la migración es principalmente masculina (Bidegain G. , 2019, p. 114).

Los datos de los Censos de los Estados Unidos de América indican que los haitianos se concentran básicamente en edad de trabajar, es decir, población económicamente activa ( $81.1 \%$ de los migrantes en 2000 y $91.7 \%$ en 2010). Por lo tanto, es fácil entender que la edad mediana es muy alta: casi 40 años en 2000 y 43.6 años en 2010. En otras palabras, hay diferentes cohortes de edad laboral que se han ido envejeciendo. La edad media en Haití es de 22 años debido al alto nivel de fecundidad en el pasado reciente.

En el 2000, los jóvenes menores de 18 años representan menos de una décima parte del total, o el $9.8 \%$, en el 2010 esa proporción baja a 8.3\%. En el año 2000 la mitad de esta población, está casada (50.7\%), a diferencia de Haití, donde predomina la convivencia o cohabitación (Bidegain G. , 2012, p. 140), esa proporción baja en el 2010 a 46.1\% aunque sigue siendo muy superior a la registrada por las diferentes encuestas tipo DHS o el Censo Población y Vivienda de 2003 de Haití (Cuadro N³).

Como se esperaba, la gran mayoría de estos migrantes son mayores de edad. Además, en el año 2000 más de 4 de cada 10 migrantes, $43.4 \%$, obtuvieron la ciudadanía estadounidense, y alrededor de uno de cada dos en el 2010; en mayor proporción las mujeres (54.2) lo cual estima "facilitaría la integración" en la sociedad de acogida. Otra vía para "incorporarse" a la sociedad de acogida es integrarse a las Fuerzas Armadas; en el censo 2000

Cuadro №3 Principales características socioeconómicas de los haitianos en EUA - 2000 y 2010

\begin{tabular}{|l|c|c|}
\hline \multicolumn{1}{|c|}{ Características } & $\mathbf{2 0 0 0}$ & $\mathbf{2 0 1 0}$ \\
\hline Población & 419,395 & 559,831 \\
\hline Masculino (\%) & 47.4 & 45.4 \\
\hline Femenino (\%) & 52.6 & 54.6 \\
\hline Edad Mediana (\%) & 39.6 & 43.6 \\
\hline Naturalizado (\%) & 43.4 & $50^{*}$ \\
& & 54.2 (Mujeres) y \\
\hline Menores de 18 años (\%) & 9.8 & 8.3 (Hombres) \\
\hline En edad de trabajar (\%) & 81.1 & 91.7 \\
\hline Estado Matrimonial & & 46.1 \\
\hline Casados (\%) & 50.7 & 1.6 \\
\hline Veteranos (\%) & 2.1 & 74.3 \\
\hline Educación & & 16.7 \\
\hline Secundaria (\%) & 13.5 & \\
\hline Licenciatura, Maestría o & & \\
\hline Doctorado (\%) & & \\
\hline
\end{tabular}

Fuente: US Bureau Census, 2000 -2010.

*Estimación ACS $^{12}$ US Bureau of Census.

12 American Community Survey (ACS) encuesta que es parte del U.S. Census Bureau. 
se registran que el $2.1 \%$ serían veteranos y $1.6 \%$ en el 2010. Otra manera sería adoptando los patrones matrimoniales de la sociedad de acogida.

Con respecto a la educación, en 2000, 3 de cada 5 migrantes, o el $61.7 \%$, alcanzaron al menos el nivel secundario y el 74.3\% en 2010. Al mismo tiempo, en Haití, el $11 \%$ ya ha alcanzado este nivel. Entre los migrantes haitianos, más de una décima parte, o el 13.5\%, tienen un título universitario (licenciatura, maestría o doctorado), mientras que solo el $1 \%$ de los haitianos que viven en Haití tienen un título universitario. En 2010, ya había un $16.7 \%$ con licenciatura, maestría o doctorado. Este nivel de educación mencionado anteriormente permite a 1 de cada 5 migrantes para el año 2000 ocupar puestos de responsabilidad en el mercado laboral y $22.6 \%$ en 2010. Es importante señalar que la finalización de la secundaria de 25 años y más es $18.3 \%$ para hombres y $18.2 \%$ para mujeres respectivamente muy superior a los niveles alcanzados en Haití.

Los hogares de inmigrantes tenían un ingreso anual promedio de US \$36,390 en el año 2000 (Bidegain G., 2012, p. 135). El ingreso promedio de los hogares de nacidos en Haití residiendo en EUA había aumentado a 40,969 US dólares en el 2010. Mientras que el ingreso per cápita era de US \$21.013. En ese momento, el ingreso per cápita en Haití era de US \$390 por año. Los hogares haitianos en Haití, cuyo tamaño era 4.6, tienen un ingreso promedio de US $\$ 1,833$, hay cada vez más mujeres jefas de hogar en Haití. Esta tendencia también refleja en los Estados Unidos donde como resultado de la migración selectiva, muchas mujeres educadas emigran a este país, como se menciona anteriormente.

Según el Censo de Población y Vivienda de Haití (2003) el 31.4\% de los hogares migrantes haitianos estaban encabezados por mujeres. En términos de nivel de ingresos, aproximadamente 1 de cada 5 hogares, o $18.9 \%$, son pobres. En el caso de Haití, en el momento de la encuesta de Condiciones de Vida (2001), el $78 \%$ vivía con menos de dos dólares al día y el $53 \%$ en pobreza extrema, es decir, con menos de un dólar por persona.

Entre los migrantes haitianos, 4 de cada 10 eran propietarios de viviendas para el año 2000 y el costo promedio de propiedad era de 122,200 dólares estadounidenses de US $\$ 213,500$ en el año $2010^{13}$ (Bidegain 2012, p. 135).
En cuanto a la situación económica, el $19.6 \%$ de los migrantes haitianos en Estados Unidos vivía por debajo del umbral de pobreza. En Haití, casi el $80 \%$ de los haitianos se encuentran en esta situación. La tasa de desempleo fue del $14.2 \%$ entre los antepasados y descendientes en los Estados Unidos. Esta tasa sigue siendo muy alta en Haití.

En relación al ingreso familiar anual promedio, fue de US $\$ 45,626$ en 2010. La falta de datos sobre este tema hace que sea imposible medir el nivel de ingresos de los haitianos y compararlo con el de los migrantes y descendientes. El nivel de ingresos de los antepasados, mencionado anteriormente, permite que $46.5 \%$ de ellos adquieran sus propias casas. La publicación de los datos del censo de 2020 para los Estados Unidos de América, desglosados por lugar de nacimiento, confirmará o invalidará estas estimaciones de la encuesta mencionada anteriormente.

\subsection{Los antepasados y descendientes de haitia- nos que viven en los Estados Unidos de América (2009)}

El censo y los últimos datos de la encuesta de antepasados migrantes (2009) muestran un aumento en el número de personas en los Estados Unidos que hablan la lengua creole haitiano en casa o tienen o tuvieron un ascendiente de ese origen.

Según el censo de 1990, estos últimos los descendientes de haitianos o personas que hablaban el creole eran 290,000 , o $0.1 \%$, de la población total. Diez años después, había 548,000 personas que hablaban creole haitiano; que representa el $0.2 \%$ de la población total (Censo 2000). En la encuesta de 2009 , esta población se estima en 830,000 , o $0.3 \%$ de la población total. Los que hablan creole haitiano se concentran particularmente en el de EUA (Florida, Nueva York, Massachusetts, Georgia, Maryland), tal como lo hacen los nativos de dicho país (Mapa $\mathrm{N}^{\circ} 2$ ).

Los antepasados y descendientes haitianos se concentran en los estados de: Florida, Nueva York, Massachusetts, Georgia, Nueva Jersey, Connecticut y Pensilvania. La situación en la región central recibe menos del $1 \%$, o el $0.8 \%$ de los migrantes (Mapa $N^{\circ} 1$ ). Es posible que esta proporción siga aumentando, particularmente en los estados antes mencionados. ¿Cuál es el nivel de integración y/o como se asimilan?; al parecer adoptan los comportamientos de la sociedad de acogida.

13 Se ha utilizado para el 2010 las estimaciones del US Bureau Census a través de la encuesta ACS 2010 de un año. 
Mapa $N^{\circ} 1$ Ancestros haitianos residiendo en Estados Unidos, año 2009

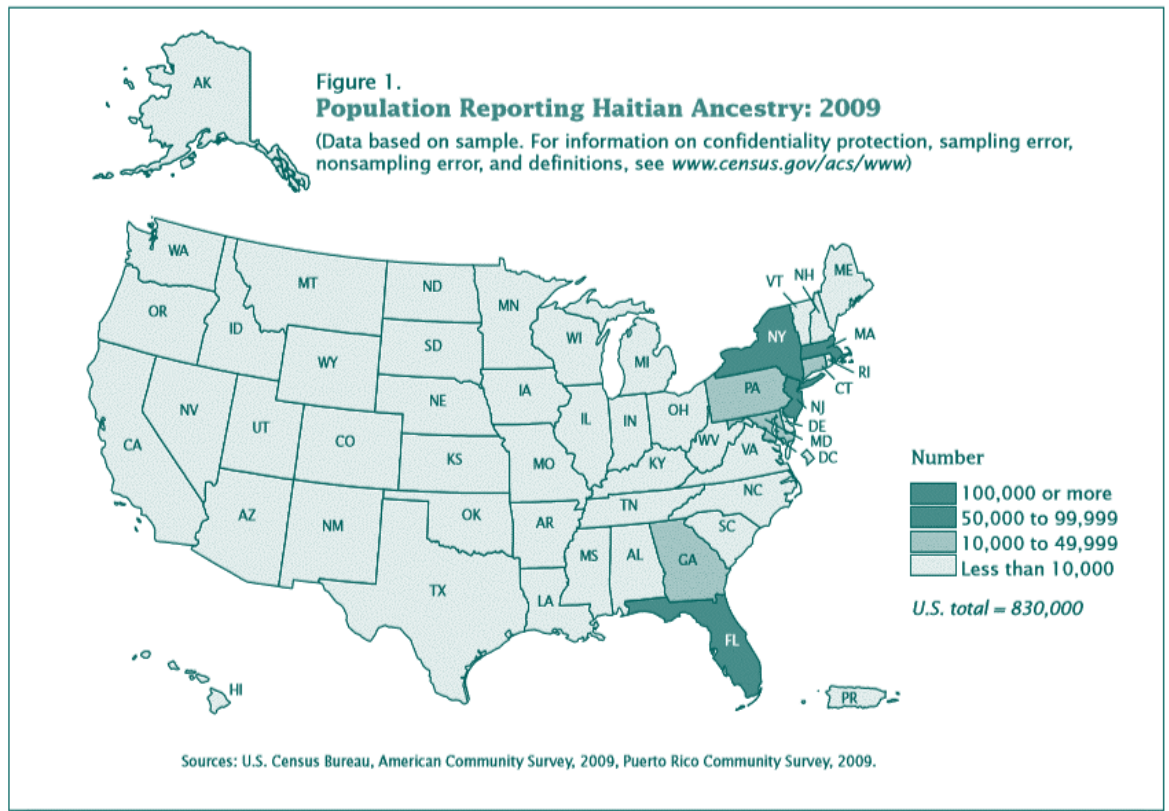

Fuente: US Bureau of Census.

Mapa $\mathbf{N}^{\circ} 2$ Los que hablan creole haitiano (no solamente nacidos en Haití)

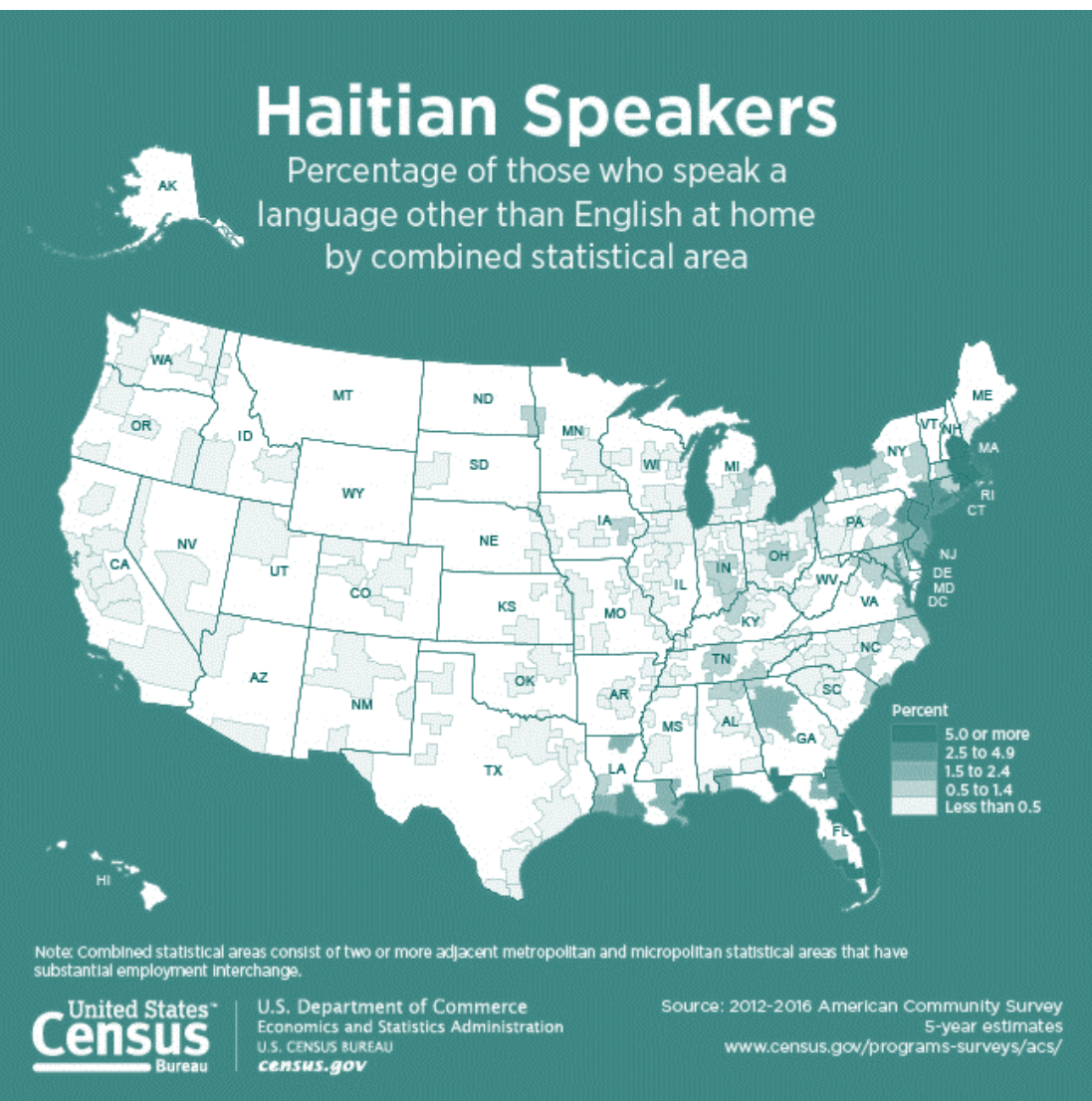

Fuente: US Bureau of Census, período 2012-2016. 


\subsection{Emigración después del terremoto del 12 de enero del 2010}

¿Cuántos se han ido al extranjero después del terremoto? Las cifras dadas son muy altas según los medios de prensa. Lo mismo a los que emigraron dentro del país. Sin embargo, debe tenerse en cuenta que también hubo una migración de retorno.

La encuesta de migración en la región metropolitana de Puerto Príncipe, comanditada por el (UNFPA - Haiti, 2010) local indica que solo el $2 \%$ de los migrantes se han ido al extranjero. En otro estudio sobre migración (Rousseau, 2010) realizado en 4 ciudades afectadas por el terremoto (Léogane, Jacmel, Petit Goâve y Grand-Goâve), el nivel fue más alto (3\%), especialmente en Jacmel, pero el volumen total de las personas (que viven en las cuatro ciudades involucradas) es muy inferior a las personas que habitan en el Área Metropolitana de Puerto Príncipe.

Durante los dos meses posteriores al terremoto del 12 de enero de 2010, todos los medios de transporte se paralizaron, a excepción de la comunicación terrestre. El principal aeropuerto internacional había sido puesto bajo el control de las fuerzas de los Estados Unidos de América como parte de la coordinación de las actividades logísticas para la ayuda estadounidense. Por tierra (Ómnibus), había 5 servicios diarios. A partir de estos datos, se puede tener una primera estimación mínima, adjunta a la estimación de aquellos que se fueron por su cuenta.

Cabe recordar que, en los primeros días después del terremoto, cruzar la frontera por Haití (vehículos privados) era casi imposible. Se estima que el número de personas que se mudaron a la República Dominicana después del terremoto no sería superior a 12,000 personas. Muchas madres de clase media decidieron (después del terremoto) enviar a sus hijos para continuar sus estudios en el extranjero, a través de la República Dominicana. Unos meses después, muchos de estos estudiantes desplazados regresaron a Haití. Sin embargo, no se sabe cuántas de estas personas desplazadas (incluidos sus hijos) han regresado.

La Encuesta de Migración del Área Metropolitana de Puerto Príncipe (AMPAP) indica que entre los residentes de AMPAP, el $20 \%$ planea ir al extranjero en los próximos 5 años (Bidegain G. , 2012, p. 137).

Al juicio EUA, habría recibido alrededor unos 10,000 individuos por año luego del terremoto muy inferior a lo que se preveía por parte de la Seguridad Americana al otorgar el TPS.
Frente a la tragedia haitiana muchos países abrieron sus puertas dentro del marco legal respectivo de cada uno de ellos. El primero fue la República Dominicana y le siguieron varios países (EUA vía el TPS, Canadá, Francia y otros). También ha habido una migración hacia países de la región como: Brasil, Chile y México utilizando varios canales para ese desplazamiento.

\section{Conclusiones}

Es necesario indicar que NO existe una sola teoría que explique las migraciones incluidas las internacionales. Además, no solo existen causas económicas (atracción) como lo indica la teórica neo-clásica sino habría una estampida (por el diferencial salarial), en particular para Estados Unidos de América todo indica que hay otros elementos que juegan o impiden en la decisión de migrar.

Todo hace pensar que los haitianos siguen buscando "al tío en EUA" tal como lo pronosticaba Price-Mars a pesar que hay una estimación de alrededor de 643,341 en 2017. La presencia haitiana en EUA sigue aumentando. Los datos del próximo Censo darán informaciones que podrán confirmar o no dichas estimaciones.

Las redes (razas/etnias) juegan un rol importante en el mantenimiento en el tiempo de la presencia haitiana en EUA. El haber decidido naturalizarse o adoptar las pautas matrimoniales debe ser un camino para "integrarse" o "asimilarse" a la sociedad de acogida.

Lo que resalta es que la migración es mayoritariamente femenina y con más alto nivel educativo. Muy diferente a la situación que se da con la migración hacia República Dominicana.

Haití tiene un potencial demográfico y expulsor importante y seguirá expulsando gente "buscando al tío" a pesar de los impedimentos legales. Es dable constatar que igual los haitianos no se van, las preguntas que surgen son: ¿es por falta de información? o ¿por impedimentos legales?, ¿falta de recursos financieros?, ¿la raza/etnicidad son un obstáculo?, ¿Cuáles son esas barreras?

Si bien se impone el estudio de los migrantes haitianos tal cual se refleja en este trabajo, además es sorprendente que incluso después del terremoto devastador del 12 de enero del 2010, solo un 2\% del Área Metropolitana de Puerto Príncipe quisiera migrar, también es cierto que en esa misma encuesta indicó que un $20 \%$ dijo que quisiera migrar en los próximos 5 años. 
Desde muy temprano del siglo pasado había inmigrantes haitianos en EUA, se conoce que bajo la dictadura de Duvalier (François y Jean Claude), la emigración era una migración intelectual. Después del golpe de estado contra el presidente Aristide en 1991, hubo una gran emigración de personas menos educadas (gente en botes-boat people) hacia diferentes destinos ver el excelente libro de SAINT - HUBERT, F. (2003). Igual ha habido una migración después del terremoto de 2010 .

Como era dable esperar los nacidos en el exterior se concentran en edad de trabajar y en plena edad fértil por lo cual muchos tienen y tendrán sus descendientes en EUA.

Cada día hay más no haitianos que hablan el creole haitiano y que siguen la distribución de ocupación territorial que tienen los nacidos en Haití. En EUA los formularios del censo son preparados en creole haitiano para esa comunidad. Lo mismo han hecho otros países de la región dado el número de nacidos en Haití que residen en dichos países (caso Chile).

Los datos del próximo Censo de Población de la ronda 2020 (primero de abril) proporcionarán más información sobre los nacidos en Haití, sus características socioeconómicas y la fecha de su llegada al país anfitrión entre otras informaciones.

\section{Referencias bibliográficas}

- ARANGO, J. (1985). Las «leyes de las migraciones» de E. G. RAVENSTEIN, cien años después. REIS, págs. 7-26.

- Bidegain, G. (2011). Los haitianos en el exterior: Mitos y realidades. En G. y. Vega Cánovas, Haití - México. Hacia una nueva política de cooperación (págs. 141-165 ). Mexico City: El Colegio de México, México.

- Bidegain, G. (2012). Les Haïtiens qui sont partis. Combien sont-ils ? Où sont- ils ? Et les transferts ? CRESFED \# 28, 132-140.

- Bidegain, G. (2019). Haití : la "invasión" a Republica Dominicana, Revista Población y Desarrollo, Universidad Nacional de Honduras, Tegucigalpa. Revista Población y Desarrollo .Argonautas y Caminantes, Universidad Nacional de Honduras, 112-117.

- Bureau of Census. (1970-2010). Census and estimation (ACS). washington, DC: US Census Bureau.

- CELADE - IMILA y US Census Bureau. (19702010). Nacidos en Haiti. Santiago de Chile, Was- hington, DC: CEPAL Y US BUREAU CENSUS.

- DOMENACH, H. .. (1998). Sobre la migratologia... . NOTAS de POBLACION, N 67-68, Año XXVI , 101-118.

- Garcia Borrego. (2006 Vol III \# 4). Generaciones sociales y sociológicas, un recorrido histórico por la literatura sociológica estadunidense sobre los hijos de inmigrantes. Migraciones Internacionales El Colegio de la Frontera Norte., págs. 5-34.

- Orozco, M. (2019). Latin American and Caribbean Migration from Weak and Failing States. Washington, DC. : Inter-American Dialogue.

- Portes, A. (2007). Migración y desarrollo: una revisión conceptual de la evidencia. Revista Mensual de Economía, Sociedad y Cultura., 21-49.

- Portes, A. (2009). Migración y cambio social: algunas reflexiones conceptuales. RES $\mathrm{n}^{-} 12$ (2009) pp. 9-37.

- Price-Mars, J. (1928). Ainsi parla l'Oncle. Essais d'ethnographie Les classiques des Science Sociales, Université de Québec (Canada), 241 p. Quebec, Canada: Université de Québec (Canadá).

- Ravestein, E. (1885). The laws of Migration. Journal of Statistics Society Royal Statistics Society Part II.

- Rousseau, J. (2010). Enquête sur les impacts du séisme et le profil sociodémographique et économique des sinistrés. Port au Prince, Haiti: Ministere de l'Economie et de Finances (MEF).

- Rousseau, J. (2019). Haïti Bilan de CIPD + 25. Port au Prince , Haïti: UNFPA.

- Saint-Hubert, F. (2003). ... Et le Neveu Répondit. AnalyseStatistique de la Migration Haïtienne aux Etats Unies (1953-2000). Trafford, Victoria, Canada:: Victoria, Canadá.

- UNFPA - HAITI. (2010). L'enquête de migration dans l'aire métropolitaine de Port -au - Prince. Port au Prince, Haïti: UNFPA.

- US Bureau Census. (2000 -2010). Census Population and ACS estimation. Washington, DC: US Bureau.

- Us Bureau Of Census. (1992). Ethnographic Evaluation Of The 1990 Decennial Census Report Series . Washington, DC: US Bureau .of Census. 